\title{
Modeling and Prediction of Krueger Device Noise
}

\author{
Yueping Guo* \\ NEAT Consulting, Seal Beach, CA 90740 USA \\ and \\ Casey L. Burley ${ }^{\dagger}$ and Russell H. Thomas \\ NASA Langley Research Center, Hampton, VA 23681 USA
}

This paper presents the development of a noise prediction model for aircraft Krueger flap devices that are considered as alternatives to leading edge slotted slats. The prediction model decomposes the total Krueger noise into four components, generated by the unsteady flows, respectively, in the cove under the pressure side surface of the Krueger, in the gap between the Krueger trailing edge and the main wing, around the brackets supporting the Krueger device, and around the cavity on the lower side of the main wing. For each noise component, the modeling follows a physics-based approach that aims at capturing the dominant noise-generating features in the flow and developing correlations between the noise and the flow parameters that control the noise generation processes. The far field noise is modeled using each of the four noise component's respective spectral functions, far field directivities, Mach number dependencies, component amplitudes, and other parametric trends. Preliminary validations are carried out by using small scale experimental data, and two applications are discussed; one for conventional aircraft and the other for advanced configurations. The former focuses on the parametric trends of Krueger noise on design parameters, while the latter reveals its importance in relation to other airframe noise components.

$=$ noise amplitude

Nomenclature

$=$ Krueger span length

$=$ Krueger chord length

$=$ directivity factor

$=$ spectral shape function

$=$ constant in bracket noise spectrum

$=$ total strut length

$=$ Mach number

$=$ source area

$=$ Strouhal number

$=$ mean flow velocity

$=$ function for Mach number dependence

$=$ power index for low frequency bracket noise spectrum

$=$ Krueger gap width

$=$ sound speed

$=$ bracket strut cross section dimension

$=$ source frequency

$=$ receiver or Doppler shifted frequency

$=$ cavity depth

$=$ power index for high frequency bracket noise spectrum

\footnotetext{
* NEAT Consulting, 3830 Daisy Circle, Seal Beach, CA 90740, AIAA Associate Fellow

$\dagger$ Senior Research Engineer, Aeroacoustics Branch, MS 461, AIAA Senior Member

* Senior Research Engineer, Aeroacoustics Branch, MS 461, AIAA Senior Member
} 


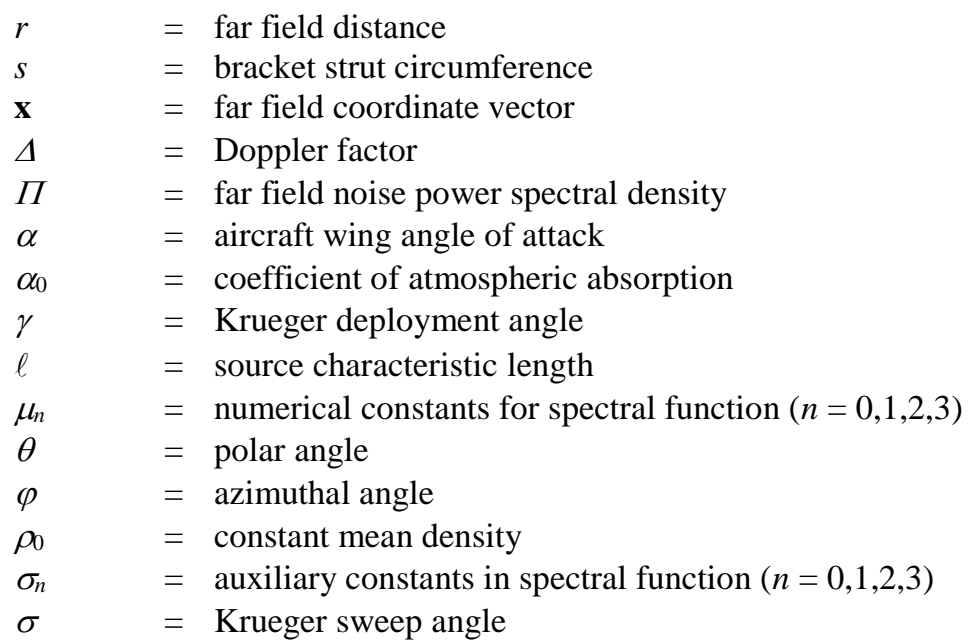

\section{Introduction}

The use of Krueger devices in high lift systems of current generation aircraft has similar benefits to conventional slotted slats in providing improved lift characteristics for landing and takeoff operations. They are considered to be even more important in future aircraft that seek to increase the extent of laminar flow or utilize flow control technologies because devices for flow control are most likely to be installed around the leading edge of the wing, making the design of slotted slats difficult. Furthermore, Krueger devices can be a shield to minimize the accretion of bugs or other debris in the flow that can negatively impact laminar flow. Therefore, Krueger flaps are considered a necessary part of high lift system design for future aircraft [Ref 1]. This, in turn, necessitates the need to include Krueger device noise in aircraft noise research [Refs 2-5]. It is this need that has motivated the work reported here; the objective of the study is to develop prediction models for Krueger device noise.

The noise generation mechanisms for Krueger devices have similarities to conventional slotted slats, but also have unique features. Flow fluctuations can exist around the Krueger devices in four regions, namely, the cove under the Krueger on the pressure side, the gap between the Krueger trailing edge and the main wing, the vicinity around the brackets connecting the Krueger to the wing, and the cavity opened up on the wing due to the deployment of the Krueger. These are illustrated in Figure 1, which shows a Krueger flap on the Boeing 747-8 aircraft [Ref 6]. In this case, the Krueger flap surface is curved, which, together with the bull nose, forms a cove on the pressure side of the device. The Krueger device can be deployed with or without gap, similar to conventional slats. The brackets and the cavity are also clearly shown in the figure. The brackets are larger than the slat tracks used on conventional slats, potentially inducing more noise, and the cavity is, of course, absent for conventional slats, and thus represents a new type of noise source for the Krueger device.

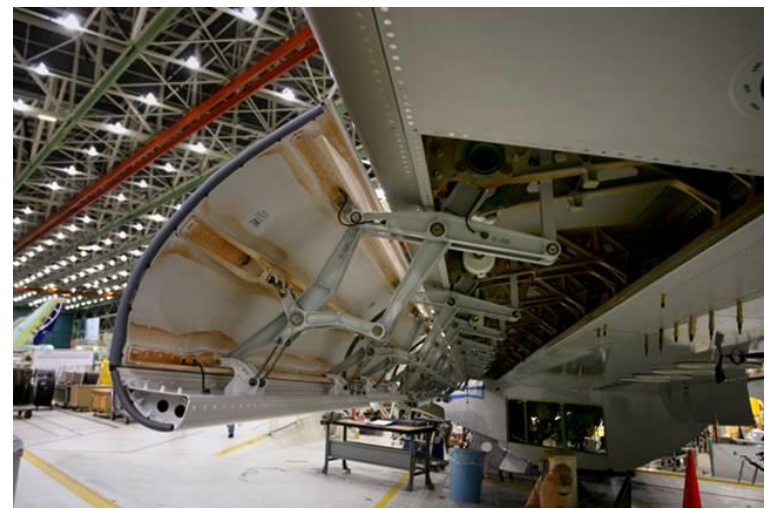

Figure 1. Krueger flap on Boeing 747-8 (from Ref 6).

The noise source mechanisms for each of the four noise components will be analyzed and their respective acoustic features discussed, and models will be developed based on the fundamental theory of aerodynamic sound generation that formulates the radiated noise in terms of source statistical properties and propagation characteristics 
[Ref 7]. Through the use of a combination of asymptotic expansion, statistical modeling and dimensional analysis, far field noise models are derived in terms of the far field noise spectrum, directivity, Mach number dependence, component amplitude, and other features. Each of these features is further developed by analytical and/or correlation analysis to relate the noise characteristics to aircraft operational and high lift system geometrical parameters. The noise from the four Krueger components is modeled individually with the total Krueger noise given by their incoherent sum, which is justified by the incoherent nature of the noise components. This is a modeling approach that has been previously used for various components of airframe noise [Refs 8-12].

To help the development of the prediction models and to provide data for validation, a wind tunnel experimental study is ongoing in parallel to the theoretical modeling effort. The wind tunnel model is a small-scale high-lift system based on the 30P30N airfoil with a Krueger flap, and the tests are being carried out in the Quiet Flow Facility (QFF) of NASA Langley Research Center [Ref 13]. Using data provided by the wind tunnel study, preliminary validation of the prediction models are shown in this paper. The results demonstrate good agreements in parametric trends, as well as noise amplitudes. This validation is only preliminary because the experimental study is still ongoing and the datasets are of very limited number. As the database expands and is refined, more thorough validations will be performed, and the prediction models will be improved as needed.

To demonstrate the application of the prediction models, two cases will be studied and presented in this paper, one for a conventional Tube-and-Wing $(\mathrm{T}+\mathrm{W})$ design and the other for a Hybrid-Wing-Body (HWB) aircraft. For the former, comparisons of noise levels will be made among four aircraft configurations. They include a turbulent wing with conventional slats, a Hybrid Laminar Flow Control (HLFC) wing with baseline Krueger flaps, an HLFC wing with aerodynamically optimized Krueger flap settings, and an HLFC optimized wing with additional noise reduction concepts. The comparisons not only reveal the acoustic effects of Krueger devices, but also bring out the relative ranking of importance of the four Krueger noise components. For the HWB application, airframe noise levels will be examined for an HWB aircraft with leading edge Krueger devices. The contributions of the Krueger flaps to the total airframe noise will be discussed and compared with other airframe components such as the landing gears and the trailing edges.

Following the introduction section, the paper will start with an analysis of the noise source mechanisms for the Krueger device in Section II. This leads to the physics-based modeling for each components, described in Section III with detailed discussions on the spectral shape and Mach number dependencies in Sections IV and V, respectively. The validations of the models will then be shown in Section VI, followed by the two applications in the two subsequent sections.

\section{Noise Source Mechanisms}

In the cove region under the pressure side surface of the Krueger, the flow can separate for some Krueger designs, especially for Krueger devices with a bull nose. In this case, the flow separation, and thus, the noise generation, is very similar to that around conventional slotted slats, which has been extensively analyzed and modeled in previous studies [Refs 8, 14-19]. For Krueger designs without a highly curved pressure side and deployed at relatively small angles, flow separation in the cove may not be very intense. Hence, the noise source mechanisms in the cove region may be mainly due to fluctuations quickly swept through the cove toward the Krueger trailing edge, producing lift fluctuations on the Krueger device. In both cases, the characteristic length scale of the noise generation process is of the same order as the Krueger chord length, which, together with the mean flow velocity, determines the peak radiation frequency of the cove noise component. Due to the approximate twodimensional nature of the source distribution, the peak radiation amplitude scales with the fifth power of the flow Mach number [Refs 8, 20-22]. Similarly to other types of flows with significant separations, the flow contains a rich array of characteristic scales, from small scales at the onset of the flow separation close to the cusp of the curved Krueger device, to the mid length scale corresponding to the shear layer instabilities, and to the large scale from the cove vortex flow oscillations. Thus, the noise can be expected to be very broadband in spectral features.

The gap noise is also similar to conventional slats and is due to the flow fluctuations passing through the high speed flow in the gap between the Krueger trailing edge and the main wing. The gap flow has features that are a combination of mass fluctuations pumping in and out of the gap and the trailing edge scattering that converts vortical energy into sound. In this case, the noise is mostly in the high frequency domain because the characteristic length scale is of the same order as the gap width. The two-dimensional source distribution and the trailing edge scattering both have a Mach number dependence of the fifth power for the overall sound levels [Refs 19, 23]. Both of these mechanisms are broadband in frequency, but due to the relatively smaller distribution of flow scales, a narrower spectral shape can be expected, in comparison with the cove flow sources. 
The bracket noise component is generated by the unsteady flows around the struts in the bracket structure, which are characterized by bluff body flow separation. Due to the irregular geometric features of the brackets, with many cutouts, steps and small details on the struts, the noise is mostly broadband and in the mid and high frequency domain, scaling on the cross-section dimensions of the struts and the sizes of the small features, respectively. The noise can be described by the theory of surface dipoles, leading to the Mach number dependence of the sixth power for the overall sound pressure. The complexity of the bracket geometry calls for statistical description of the noise mechanisms, similar to that used in aircraft landing gear noise modeling [Refs 9, 10], which scales the noise amplitude on both the total surface area of the struts and a complexity factor that accounts for the effects of small details in the bracket assembly. In the extreme of clean struts, the noise radiation would be mostly tonal, corresponding to the regular vortex shedding from the struts. The irregularity of the strut geometry suppresses the pure tone generation, but the broadband spectrum for this noise component can still be expected to be narrow in shape.

The cavity noise component associated with Krueger devices is due to separated flows around and inside the cavity, which opens when the Krueger flap is deployed. Though cavity noise has been quite extensively studied in the past, especially its tonal component, there have not been sufficient data and research on the noise mechanisms for the Krueger cavity, which has a few unique features that may define its noise characteristics. First, the incoming flow just upstream of the cavity is extremely turbulent, unsteady and non-isotropic, due to the cove flow separation and the wake of the supporting brackets. This is different from cavities with an attached boundary layer as incoming flow, which develops a shear layer over the cavities that is necessary for the tonal noise generation commonly studied in the past. Secondly, there are many mechanical components and irregular geometric features inside the cavity so that the Krueger cavity does not have a regular shape and volume. Because of these features, the pure tone generation due to a shear layer/acoustic feedback is not expected. The noise source is probably dominated by the vortex rollup around the leading edge of the cavity, which resembles a backward facing step. The unsteady vortex rollup is similar to the flow around the flap side edges studied in Ref 11, in which case, the noise is broadband.

\section{Prediction Model}

Airframe noise prediction models have previously been developed [Refs 8-12], based on the fundamental theory of aerodynamic sound generation [Ref 7], for all the major airframe noise components, including the leading edge slats, the flap side edges, the trailing edges, and the landing gears. As discussed in the previous section, the noise source mechanisms of the Krueger noise have similarities to other airframe noise components, and thus, the methodologies developed in those studies can be readily carried over for the use of Krueger noise prediction. Thus, each of the four Krueger noise components can be formulated in terms of the power spectral density of the far field noise, denoted by $\Pi$, which has the general form

$$
\Pi(\mathbf{x})=\rho_{0}^{2} c_{0}^{4} A(\alpha, \gamma, \sigma, b, S) W(M) F\left(f_{d}, M\right) D(\theta, \varphi) \frac{S}{\Delta^{4} r^{2}} e^{-\alpha_{0} r} .
$$

In this solution, the major dependencies of the far field noise on various operational and geometrical parameters are separated from each other. These dependencies are summarized in Table 1, where the physical features of the far field noise are listed in the first column, their respective mathematical models are shown in the second column, and the approaches to derive these functional dependencies are explained in the last column. It should be pointed out that though the general expression of the far field noise, as given by Equation (1), can also be obtained through an empirical modeling approach, it is derived here from theory (as reported in Refs 8-12). This approach not only leads to the general expression, but also explicitly results in the formulas for the individual functional dependences. These will be discussed in detail in the subsequent sections.

In the general solution given by Equation (1), the far field microphone is specified by its coordinate vector $\mathbf{x}$, defined by

$$
\mathbf{x}=\left(x_{1}, x_{2}, x_{3}\right)=r(\cos \theta, \sin \theta \cos \varphi, \sin \theta \sin \varphi),
$$

where the first representation is in Cartesian coordinates and the second is in spherical coordinates with the polar angle denoted by $\theta$ and the azimuthal angle by $\varphi$. The coordinate system is illustrated in Figure 2, where the flight direction is defined in the positive $x_{1}$-direction which is also the direction of $\theta=0$. The azimuthal angle $\varphi$ is defined such that the space under the aircraft corresponds to $\varphi$ from $180^{\circ}$ to $360^{\circ}$, and the overhead location under the flight path is $\varphi=270^{\circ}$. The microphone distance $r$ is simply given by

$$
r=|\mathbf{x}|
$$


with the vertical bars denoting modulus. The microphone distance $r$ appears in the general solution (1) in the factor $1 / r^{2}$, resulting from the spherical spreading of the sound waves as they propagate away from their sources. The propagation also suffers from atmospheric absorption, modeled in the solution as an exponentially decaying multiple of the noise power spectral density, with $\alpha_{0}$ being the absorption coefficient.

Table 1 Functional dependencies of Krueger noise model.

\begin{tabular}{lll}
\hline Feature & Model & Modeling Approach \\
\hline Ambient Medium & $\left(\rho_{0} c_{0}^{2}\right)^{2}$ & Dimensional Analysis \\
Amplitude & $A(\alpha, \gamma, \sigma, b, S)$ & Correlation \\
Mach Number & $W(M)$ & OASPL Scaling \\
Spectral Shape Function & $F\left(f_{d}, M\right)$ & Source Statistics \\
Doppler Shift & $f_{d}$ & Analytical \\
Directivity & $D(\theta, \varphi)$ & Source Integration \\
Source Dimension & $S$ & Dimensional Analysis \\
Convective Amplification & $\Delta^{4}$ & Analytical \\
Spherical Spreading & $r^{-2}$ & Analytical \\
Atmospheric Absorption & $e^{-\alpha_{0} r}$ & Empirical \\
\hline
\end{tabular}

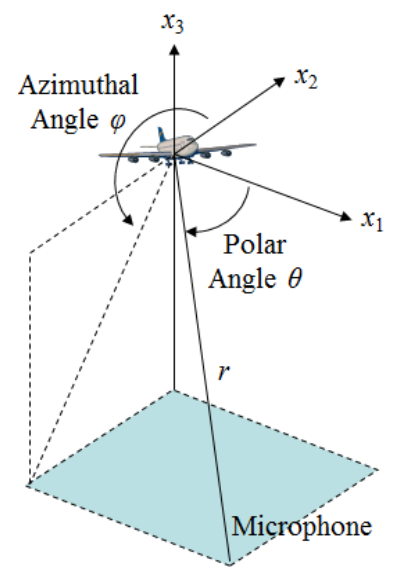

Figure 2. Coordinate system definition of the flying aircraft.

In Table 1 and Equation (1), the ambient medium is characterized by the constant mean density $\rho_{0}$ and sound speed $c_{0}$, the combination of which scales the noise power spectral density. This is derived from dimensional analysis. Also derived from dimensional analysis is the effect of source dimension, denoted by $S$. The definition of this surface area quantity depends on the nature of the sources. For the cove flow, it is of the same order as the Krueger surface area, approximately equal to the product of the Krueger chord $C$ and its span length $B$. For the gap flow, it can be approximated by the product of the Krueger span length and the gap width $b$. For the Krueger brackets, the source dimension scales on the total surface area of the struts, given by the circumference $s$ times the total length $L$. For the cavity noise sources, the source area can be estimated as the product of the Krueger span and the cavity depth $h$. This is summarized in Table 2 .

As indicated in the general solution, the noise amplitude is denoted by $A$ and is a function of other parameters, such as the aircraft angle of attack $\alpha$, the Krueger deployment angle $\gamma$, the wing leading edge sweep angle $\sigma$, and the gap width $b$. The relation between the noise amplitude and these parameters is derived by correlation analysis. It can be noted that the surface quantity $S$ has already been included in the noise model to scale the effects of source dimensions. The source dimensions are also included in the expression for $A$ because variations in the geometry may also change the source strengths, in addition to the source size. It should also be pointed out that Krueger noise can be significantly affected by other parameters not included in the above expression if the Krueger device is designed by advanced noise reduction considerations. For example, the contour of the Krueger cove may be designed to minimize noise radiation, which represents local geometrical changes not included in the general expression. 
Table 2 Definition of the source dimension $S$.

\begin{tabular}{ccc}
\hline Noise Source & $\begin{array}{c}\text { Source } \\
\text { Dimension }\end{array}$ & Definition \\
\hline Cove Flow & $C \times B$ & Krueger Chord $\times$ Krueger Span \\
Gap Flow & $b \times B$ & Gap Width $\times$ Krueger Span \\
Bracket Flow & $s \times L$ & Strut Circumference $\times$ Total Length \\
Cavity Flow & $h \times B$ & Cavity Depth $\times$ Krueger Span \\
\hline
\end{tabular}

The noise amplitude also depends on the flow Mach number $M$, defined by the flight velocity $U$ divided by the sound speed $c_{0}$, but that functional relation is accounted for by the quantity $W$, which can be found by the scaling laws of various types of sources. It should be pointed out that the conventional simple power laws for the Mach number dependence are not assumed here. As discussed in Refs 8-12, the integer power law is only suitable for scaling the overall noise levels. Airframe noise test data have not been successfully scaled on a single integer power law, at least not uniformly in all the frequencies of practical interest in aircraft noise (e.g. Ref 19), strongly indicating that the flow Mach number is an independent parameter in the spectral shapes, in addition to being a parameter to normalize the frequencies to Strouhal number. This is probably due to the presence of multiple sources in the flows, and the sources may have different Mach number dependencies. Thus, the dependence of noise on the Mach number is partially captured by the spectral shape function, as modeled by the function $F\left(f_{d}, M\right)$, and the modeling of the total Mach number effect needs to take this into account.

The frequency, denoted by $f$, and the flow Mach number both appear in the function $F$ that defines the spectral shape of the noise component. It is represented as a function of the Doppler shifted frequency $f_{d}$ defined as

$$
f_{d}=f / \Delta,
$$

with $\Delta$ being the Doppler factor as conventionally defined by

$$
\Delta=1-M \cos \theta .
$$

The Doppler factor also appears in the model (1) as an inverse fourth power. Equations (4) and (5) are, respectively known as the frequency shift and amplitude amplification, due to the effects of moving sources. The Doppler effects of frequency shift and amplitude amplification involve the polar angle, and thus are also part of the overall directivity of the far field noise. The rest of the directivity results from the nature of the sources, which, in the case of Krueger noise, is dominantly of dipole nature, due to the low flow Mach number, and is denoted by $D$ as a function of the polar and azimuthal angle.

\section{Spectral Shape Function}

In modeling aircraft slat noise in Ref 8 , detailed derivations are given for the spectral shape function $F$, by modeling the effects of sound propagation, the temporal coherence of the source distribution, and the spatial coherence of the sources. The methodology can be followed here for the application of Krueger noise. Without repeating the mathematics, the result can be quoted as

$$
F\left(f_{d}, M\right)=\frac{M^{2} S t^{2}}{\left(1+\mu_{0}^{2} S t^{2}\right)\left(1+\mu_{1}^{2}(1+M)^{2} S t^{2}\right)\left(1+\mu_{2}^{2} M^{2} S t^{2}\right)\left(1+\mu_{3} M S t\right)},
$$

where in addition to the flow Mach number $M$, the spectral function also involves the source flow Strouhal number $S t$, defined by

$$
S t=f_{d} \ell / U
$$

which scales on the source characteristic length $\ell$ and the mean flow velocity $U$. The set of quantities $\mu_{i}$ with $i=0,1,2,3$ are empirical constants. The source characteristic length scale can be estimated according to the nature of the sources for the four Krueger noise mechanisms. This is summarized in Table 3, which shows that the length scales are the Krueger chord length, the gap width, the strut cross section size and the cavity depth, respectively, for the four sources.

To illustrate the features of the spectral shape function, the result (6) is plotted in Figure 3, as a function of the flow Strouhal number at various values of the flight Mach number. The spectra are clearly broadband, consistent 
with experimental observations for Krueger noise. It can be seen that the Strouhal number scaling aligns the peak Strouhal numbers at various Mach numbers approximately at Strouhal number of unity, but the levels deviate from each other significantly at low frequencies. The figure clearly demonstrates that the spectral shapes of the Krueger noise depend on the mean flow Mach number, and there does not seem to be a simple scaling that can eliminate the explicit Mach number dependence. Physically, it is a manifestation of the complex source mechanisms and the complex radiation processes.

Table 3 Definition of the source characteristic length $\ell$.

\begin{tabular}{ccc}
\hline Noise Source & $\begin{array}{c}\text { Characteristic } \\
\text { Length }\end{array}$ & Definition \\
\hline Cove Flow & $C$ & Krueger Chord \\
Gap Flow & $b$ & Gap Width \\
Bracket Flow & $d$ & Strut Cross Section Size \\
Cavity Flow & $h$ & Cavity Depth \\
\hline
\end{tabular}

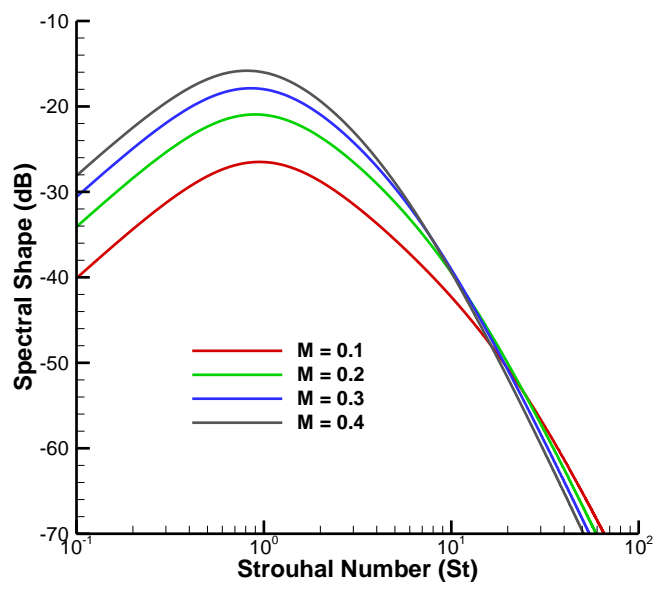

Figure 3. Spectral shape functions at various Mach numbers.

As detailed in Ref 8, the physical mechanism for the spectral shape function (6) is the unsteady pressure fluctuations on the surfaces of the high lift system bounding the noise generating flows, together with the characteristics of noise propagation from elongated source distributions approximated by two-dimensional sources. Clearly, the mathematical derivations are applicable to the cove flow for the Krueger device, the gap flow between the Krueger trailing edge and the main wing, and the cavity flow opened up by the deployment of the Krueger device, because all of them are adjacent to elongated geometry, and are known to generated noise dominantly by surface pressure fluctuations at low flow Mach numbers. The applications of the spectral shape function (6) to the three respective source flows differ from each other only in the definitions of characteristic length of the source process, as given in Table 3, and in the values of the empirical constants.

For the noise component associated with the Krueger brackets, the noise sources are still the surface pressure fluctuations on the struts of the bracket structure, but the statistical properties of the surface pressures are different from the other three components, due to the very different geometry of the bracket, compared with the Krueger device and its cavity. The source mechanisms in this case are similar to those in landing gear noise generated by the main struts and the small details attached to the main struts in the gear assembly. As analyzed in Refs 9 and 10, the sources are compact compared with the strut cross sections and are statistically homogeneous in the longitudinal direction of the struts. The noise generated by this type of source is in the mid to high frequency domain, and its spectral shape function can be represented as 


$$
F(S t)=\frac{S t^{a}}{\left(H+S t^{\mu}\right)^{q}},
$$

where the indices $a, \mu$ and $q$ are empirical constants that jointly define the spectral shape of the normalized spectrum; $H$ is a parameter to ensure that $F$ assumes its maximum of unity at a given value of the Strouhal number. It can be noted that the spectral function for the Krueger bracket noise does not contain the flow Mach number, and the effects of the Mach number on this noise component are accounted for mainly by the Mach number dependence $W(M)$.

The spectral shape functions for the four Krueger noise components are shown in Figure 4, plotted as a function of the flow Strouhal number and normalized to unity peak value. All curves are for $M=0.2$, except for the bracket noise spectrum which is Mach number independent. All four components are broadband, peaking at unity Strouhal number. This determines the dominant frequency by the flow velocity and the characteristic length of the source process. The rates of falloff on both sides of the spectral peak, or the width of the spectral shape functions, reflects the different length distribution of the sources. Figure 4 shows that the cove flow, represented by the red curve, has the broadest spectrum, resulting from the richness of the scales in the cove flow. In comparison, the spectrum for the gap flow, given by the green curve, falls off more rapidly for Strouhal numbers away from the peak value. This is to model the relatively fewer source scales in the gap flow, which is basically dominated by the gap width. The bracket noise, given by the blue curve, has even narrower spectral shape because the noise generation in this case mostly results from the vortex shedding from the struts and scales on the cross dimensions of the struts. The fourth component, the cavity noise, is represented in the figure by the gray curve and can be seen to have a spectral width similar to the gap flow.

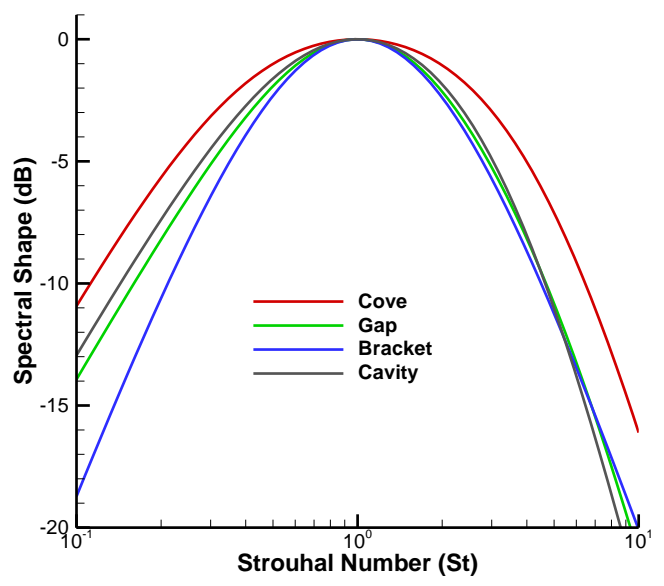

Figure 4. Component spectral shape functions.

\section{Mach Number Dependence}

The Mach number dependence of the Krueger noise components can be derived by using the scaling laws of the overall sound levels. This is the approach discussed in detail in Refs 8-12 for various airframe noise components. The approach is also applicable to Krueger noise so that the results are quoted here as

$$
W(M)=M^{2} / I(M),
$$

where $I$ is an auxiliary function given by

$$
I(M)=\sum_{n=0}^{2} \frac{\sigma_{n}^{2}}{\sigma_{3}^{2}+\sigma_{n}^{2}}\left[\sigma_{n} \frac{\pi}{2}+\sigma_{3} \ln \frac{\sigma_{3}}{\sigma_{n}}\right] / \prod_{\substack{m=0 \\ n \neq m}}^{2}\left(\sigma_{m}^{2}-\sigma_{n}^{2}\right),
$$

for the cove, gap and cavity noise component and by

$$
I(M)=M^{-4},
$$


for the bracket noise component. Here, a set of constants has been introduced to save writing and they are defined by

$$
\sigma_{0}=\mu_{0}, \quad \sigma_{1}=\mu_{1}(1+M), \quad \sigma_{2}=\mu_{2} M, \quad \sigma_{3}=\mu_{3} M .
$$

Thus, the combined model that includes the effects of both Mach number and spectral shape assumes the form

$$
W(M) F\left(f_{d}, M\right)=M^{2} F\left(f_{d}, M\right) / I(M) .
$$

This result models the Mach number effects of the power spectral density of the Krueger noise.

To illustrate the features of the Mach number dependence, the result (13) is plotted in Figure 5 in terms of the $1 / 3$ octave band levels at various Mach numbers for the cove noise component. The noise levels are plotted as a function of the flow Strouhal number, with the results scaled by the fifth power law. It can be seen that the Strouhal number scaling aligns the spectral peak frequency well, and the spectral peaks are a slight decreasing function of the flow Mach number. In comparison with the narrowband results shown in Figure 3 and Figure 4, the locations of the spectral peaks in terms of the $1 / 3$ octave band levels, however, have shifted upwards to about two, due to the $1 / 3$ octave integration. The figure also shows that the spectral shapes at different Mach numbers are not a simple shift in amplitude, as the amplitude scaling plotted in the figure does not collapse the curves uniformly in Strouhal number. While the low frequency part of the spectra seems to follow the fifth power law, significant scatter is seen at mid and high frequencies.

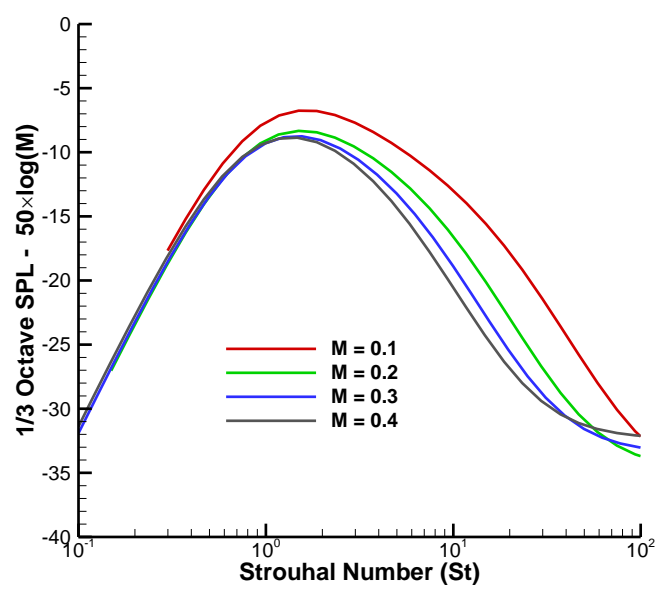

Figure 5. Mach number effects for the cove noise component.

\section{Preliminary Validation}

Noise generation by Krueger devices and its impact on the total aircraft noise has been attracting attention in acoustics research only recently, due to the potential use of such devices in future aircraft with flow control technologies. Thus, there is a scarcity of experimental data. To help the prediction model development and to provide data for tool validation, a parallel effort has been carried out also under the NASA Environmentally Responsible Aviation (ERA) project, in which a two-dimensional Krueger flap model has been tested in the NASA Quiet Flow Facility (QFF). The test model and the test setup are described in detail in Ref 13. The experimental study is still ongoing, and only some preliminary data are currently available for tool validation.

Figure 6 shows the Mach number dependence of the Krueger noise, with the curves being the predictions and the symbols being the test data from QFF. The various symbols represent various polar angles, ranging from 54 to 125 degrees in the flyover plane, where zero degrees is the flight or upstream direction. The data are as measured, and the predictions are for the QFF test setup and model geometry without any extrapolation. It is clear from the figure that the Mach number dependence of the Krueger noise is well modeled and predicted, approximately following the fifth power law due to the approximately two-dimensional nature of the sources. The QFF test model is towdimensional so that there is no sweep angle effects. The test setup does not have any brackets, which would furnish three-dimensional sources and generate noise scaling on the sixth power law on Mach number. Even in the case where the brackets are included, the bracket noise component will be in high frequencies, while the spectral peak that dominates the overall noise levels is at low frequencies, mostly resulting from the cove and cavity noise 
component. Thus, the Mach number dependence of the overall sound pressure levels can still be expected to scale approximately on the fifth power.

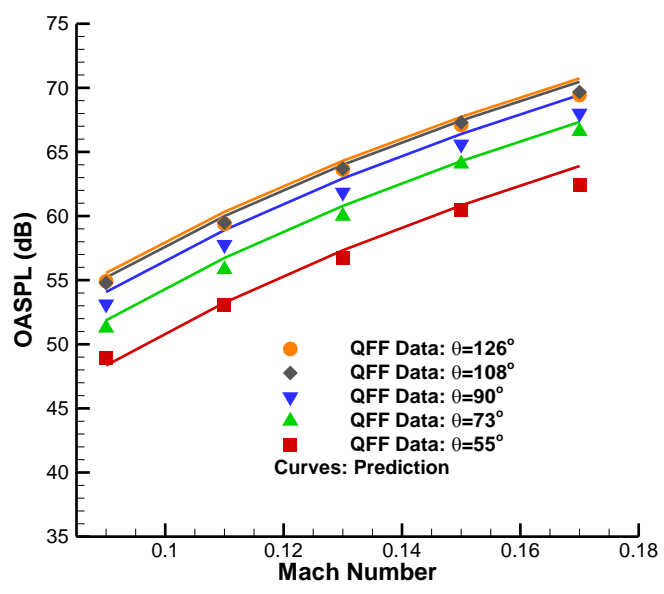

Figure 6. Mach number dependence of Krueger noise.

The data and predictions shown in Figure 6 are plotted again in Figure 7 as a function of the polar angle at various Mach numbers. The good agreements between the predictions and data are again clearly shown. Figure 7 explicitly demonstrates the directivity patterns of the Krueger noise. Similar to slat noise, the far field radiation patterns for Krueger noise are broad with gradual variation with the polar angle. The radiation peaks are in the aft quadrant because of the orientation of the Krueger flaps.

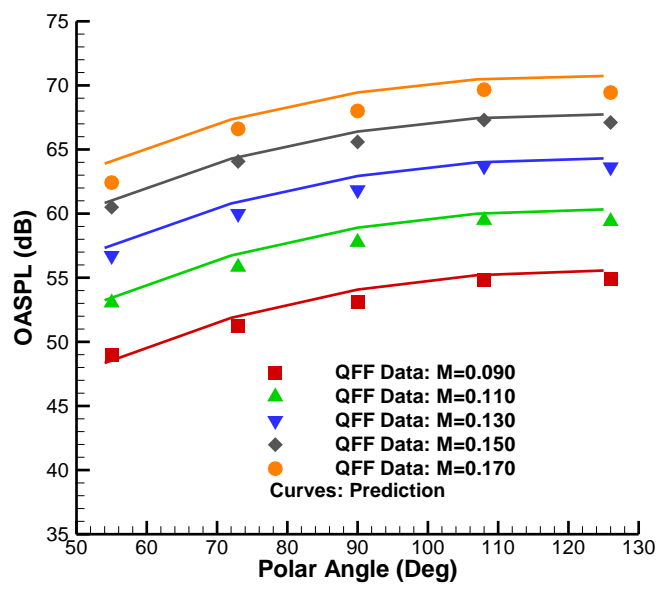

Figure 7. Directivity of Krueger noise.

To demonstrate the accuracy of the predictions for the sound pressure spectrum, Figure 8 plots some examples of the comparison between predictions and measured data. The spectra shown in the figure are at 90 degrees emission angle in the flyover plane for various Mach numbers. The predictions are for the QFF test configuration. The frequencies are high because of the small scale of the model. Similar to slat noise, the spectra are broadband. 


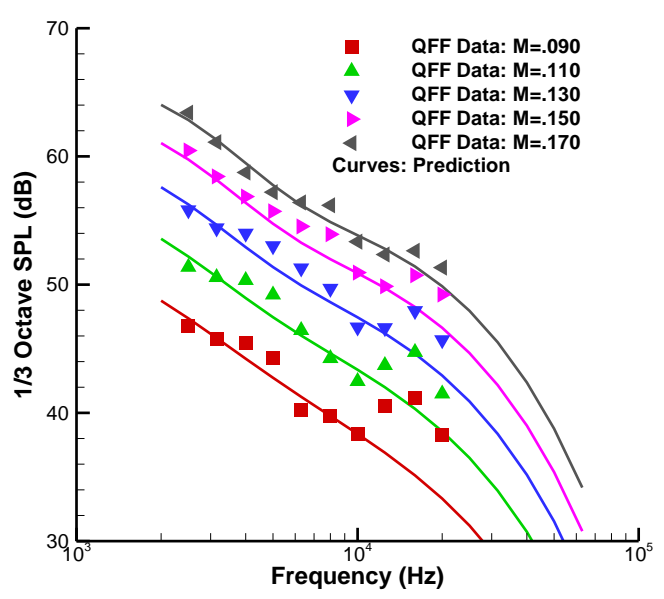

Figure 8. Spectra of Krueger noise at 90 degrees emission angle.

\section{Application to $\mathbf{T}+\mathrm{W}$ Aircraft}

In comparison with conventional slats, Krueger devices usually have a larger chord length, designed so as to ensure the required lift, especially at larger angles of attack when maximum lift is needed. This large chord length also exposes a large cavity in the main wing, as can be clearly seen in Figure 1. The cavity contains various structural parts of very irregular shapes and dimensions. The large Krueger devices need an array of brackets for support. As is seen from Figure 1, these brackets are massive in size, and their components are irregular in shape. All of these characteristics are potential noise sources, which leads to the question of relative noise levels of the Krueger devices, compared with conventional slats. It should be pointed out upfront in discussing the comparisons between Krueger flaps and slotted slats that the comparisons are relevant only when the two are designed and discussed with their respective wings, because there is no equivalence between the two in isolation. The use of one, instead of the other, is dictated by the overall high lift system design, and as such, the comparisons of the noise levels of the two need to be considered under the respective high lift systems, which may differ not only in the leading edge devices, but also in other geometric and operational parameters.

To this end, this section discusses an example of the application of Krueger devices, namely, their use in Hybrid Laminar Flow Control (HLFC) wings, where they replace the conventional slats to serve as elements of the high lift system for improved lift characteristics, and also to form a shield for the main element leading edge. The flow control technology itself is not of concern here; the focus is the change of relative noise levels when the application of such a technology leads to the use of Krueger flaps and the corresponding modifications of the high lift system. Thus, a series of four aircraft configurations is considered here: the turbulence wing, the HLFC wing, the optimized HLFC wing, and the optimized low noise HLFC wing. Their precise definitions are discussed in the following paragraphs, and their features which affect the Krueger noise levels are summarized in Table 4.

Table 4. Comparison of features of four wing designs.

\begin{tabular}{ccccc}
\hline & $\begin{array}{c}\text { Case 1 } \\
\text { Turbulence } \\
\text { Wing }\end{array}$ & $\begin{array}{c}\text { Case 2 } \\
\text { Baseline } \\
\text { HLFC Wing }\end{array}$ & $\begin{array}{c}\text { Case 3 } \\
\text { Optimized } \\
\text { HLFC Wing }\end{array}$ & $\begin{array}{c}\text { Case 4 } \\
\text { Low Noise } \\
\text { HLFC Wing }\end{array}$ \\
\hline AOA (Deg) & 6 & 4 & 4 & 4 \\
Deployment Angle (Deg) & 34 & 64 & 34 & 34 \\
Wing Sweep (Deg) & 35 & 25 & 25 & 25 \\
Chord (\% of Case 1) & 100 & 100 & 120 & 120 \\
Gap (\% of Case 1) & 100 & 100 & 100 & 50 \\
Leading Edge Device & Slat & Krueger & Krueger & Krueger \\
Cavity & No & Open & Open & Closed \\
Bracket & Normal & Normal & Normal & Aligned \\
\hline & \multicolumn{3}{c}{}
\end{tabular}


The starting point is a conventional turbulence wing with slotted slats, corresponding to designs in current generation of aircraft and serving as the reference for the comparisons. As can be seen from Table 4, the parameters that affect the noise from the leading edge device are the operational conditions such as the aircraft angle of attack and the slat/Krueger deployment angle, the dimensions of the device such as its chord length, gap width and sweep angle, and noise reduction concepts such as closing the cavity and aligning the bracket with the flow.

The second configuration is an HLFC baseline wing, which simply replaces the slotted slat with Krueger flaps of the same chord length. Due to the use of the HLFC technology, this configuration will, however, differ from the conventional wing in some other aspects, in addition to the different leading edge devices. Noticeably, there is a cavity for the HLFC wing, associated with the deployment of the Krueger flaps. The HLFC wing also has less sweep to facilitate the laminar flow. It is known that when a slat is replaced by a Krueger of the same chord length, the lift characteristics of the high lift system degrade, both in the total lift at normal operation conditions and in the maximum lift at large angles of attack for emergency operations. To compensate, the Krueger is usually deployed at a larger angle than an equivalent slat, which brings up the total lift at normal operations. The HLFC wing is also operated at a smaller angle of attack than the conventional wing, because of the lower value of maximum lift. By aircraft noise certification regulations, noise flight testing is done at a fixed percentage of the maximum lift, meaning that the smaller the maximum lift, the smaller the angle of attack for the noise test and prediction. This is beneficial to noise because smaller angles of attack usually mean less noise, but the design may not be aerodynamically acceptable due to the lower maximum lift.

Thus, even though the baseline HLFC wing is of interest in comparing the change in noise levels, it should not be considered a viable design in practical applications. Instead, it is likely that an HLFC wing will be optimized for aerodynamics so that the maximum lift is comparable to the conventional wing, which is a requirement in safety certification. The optimized HLFC is the third configuration considered here. Without going into the details of aerodynamic optimization, the feature of an optimized HLFC wing that affects the noise most is the chord length of the Krueger. Thus, a larger chord is assumed for the aerodynamically optimized configuration here for noise calculation.

The configuration optimized for aerodynamics does not, however, necessarily mean low or minimum noise. To achieve low noise, the fourth configuration includes some noise reduction concepts such as aligning the brackets with the mean flow, reducing the gap width, and closing the cavity. By conventional design, the supporting brackets for slats and Krueger devices are normal to the leading edge of the wing, which is the most convenient way for structural design and operation. Because of the wing sweep angle, this puts the bracket assembly at an angle to the incoming flow, increasing the flow separation and vortex shedding behind the struts, and thus increasing the noise generation. This acoustic disadvantage is known to be easily amendable by aligning the bracket with the flow [Ref 18]. Aligned brackets are considered here as a noise reduction concept, with the understanding that their practical implementation needs to overcome difficulties in structural and operational design. This concept also applies to slotted slats.

Before comparing the noise levels of the four configurations, it is instructive to first examine the noise components of the individual configurations because the unique features of each configuration usually affect mostly one or two noise components. For the baseline HLFC wing, Case 2 shown in Table 4, the Krueger noise decomposition is shown in Figure 9. The component-specific and total 1/3 octave band sound pressure levels are shown as a function of frequency for the emission angle of 90 degrees in the aircraft flyover plane. It is clear from this figure that the cove flow and the cavity flow contribute mostly in the low and mid frequency domain, while the high frequency noise is dominantly given by the brackets and the gap flow. It is also clear that though there are differences in the component amplitudes, all four components make noticeable contributions to the total Krueger noise, in their respective frequency domains. 


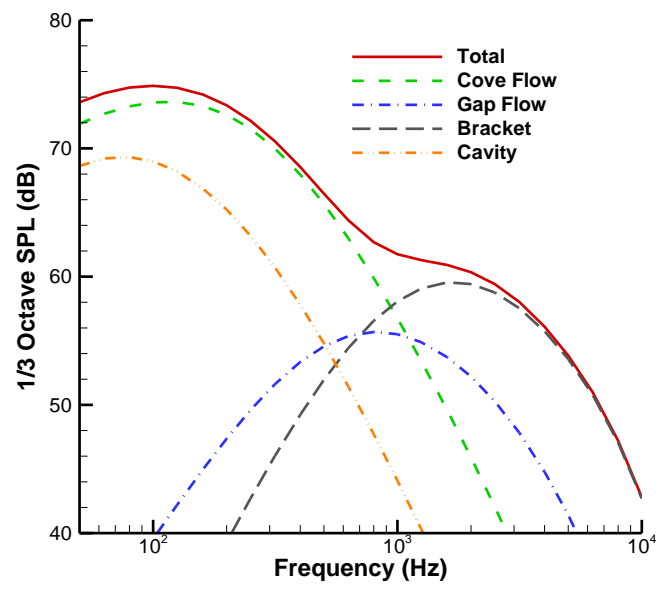

Figure 9. Components of Krueger noise for HLFC wing.

The noise levels of the four configurations listed in Table 4 are shown in Figure 10 for the emission angle of 90 degrees in the aircraft flyover plane. The solid red curve shows the sound pressure level for the conventional wing with slotted slats. When this wing is replaced by a baseline HLFC wing, the noise in the mid frequency band is reduced by about $3 \mathrm{~dB}$, as shown by the dash green curve, mainly due to the reduced flow separation in the Krueger cove region. There is an increase in noise in the low and high frequency domain, because the cavity flow generates low frequency noise and the brackets increase high frequency noise. The latter results from the larger and more complex structures of the Krueger brackets. The next case plotted in Figure 10, the HLFC wing optimized for aerodynamics, is represented by the dash-dot blue curve. In this case, the Krueger chord length is increased so that the peak frequency shifts lower. There is, yet again, some noise increase in the high frequency domain, from the dash green curve to the dash-dot blue curve in the figure. This increase is due to the increased lengths and dimensions of the brackets, necessary to support the larger Krueger flaps. The decrease in cove and cavity noise for the optimized HLFC wing is due to the lower Krueger deployment angle. The fourth configuration listed in Table 4, namely, the HLFC wing with noise reduction technologies, is represented in the figure by the long-dash gray curve. The noise reduction measures include closing the cavity, reducing the Krueger gap width, and aligning the brackets with the mean flow. The first reduces the low frequency noise, while the last two reduce the mid and high frequency noise, respectively. These are clearly shown in Figure 10.

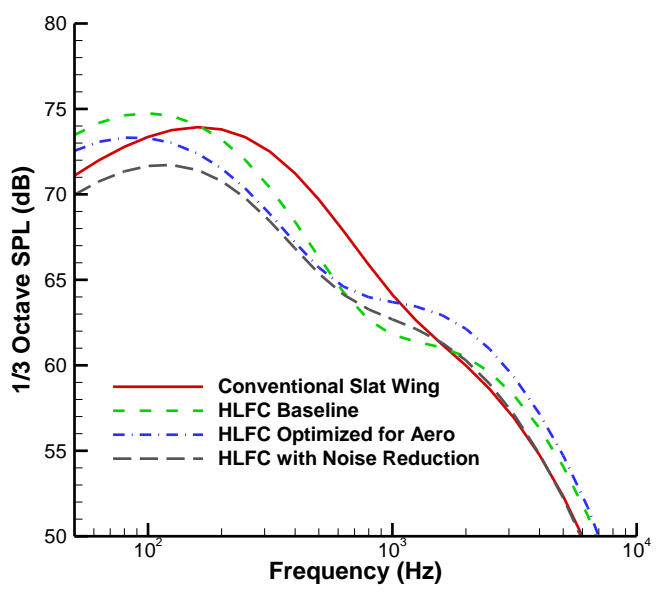

Figure 10. Noise level comparison of leading edge devices for various wing configurations.

To further compare the noise levels of the four configurations with consideration of their far field directivities, duration effects, spectral features and other factors, the Effective Perceived Noise Level (EPNL) for the four 
configurations, as well as their respective component levels, are shown in Figure 11. A few interesting conclusions can be drawn from this figure. The first is the total EPNL for the leading edge devices. It can be seen that unless noise reduction treatments are applied, the noise levels are relatively unchanged from the slotted slats to the Krueger flaps. This reflects the physical design requirement that all the wings have comparable aerodynamic performance. Though there is no quantitative design in the study, the changes from one configuration to another have followed the general qualitative guidelines to maintain the aerodynamic properties by measures such as changing the device size and deployment angle. From Table 4, it is clear that each configuration change involves more than one parameter. Thus, a configuration change is always accompanied by noise changes of more than one component, and the changes in noise levels due to these parameters are not necessarily all increasing or all decreasing. This leads to the mutual compensation of the individual noise changes and leads to the relatively unchanged total noise levels.

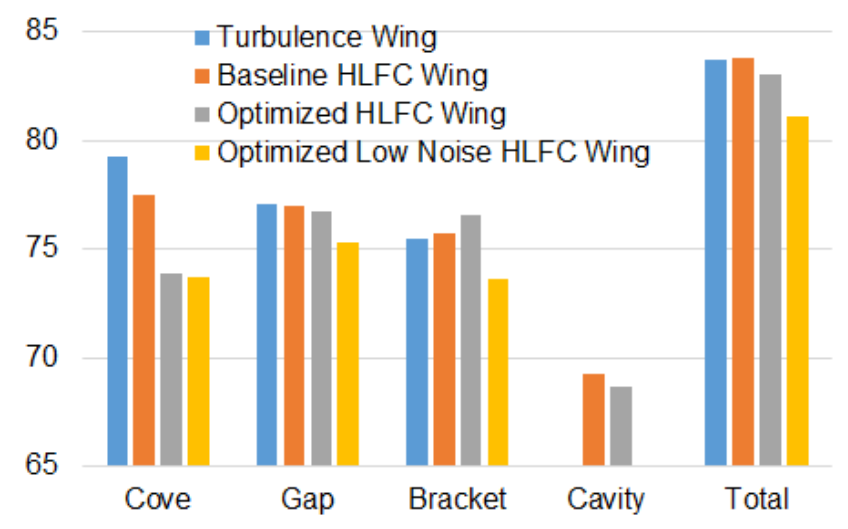

Figure 11. Noise levels of leading edge devices.

The component noise levels shown in Figure 11 more closely track the individual changes to the wing configurations. For the cove component, the highest noise level is the slotted slats that support intense flow separation in the cove region due to the highly curved surface contour on the pressure side of the slat. This noise is reduced by almost $2 \mathrm{~dB}$ when the slats are replaced by Krueger flaps because of the reduced flow separation in the cove region, even though the Krueger flaps are deployed at higher angles to maintain the aerodynamics. The optimized HLFC wing qualitatively achieves the desired performance by increasing the Krueger size while reducing its deployment angle, which reduces the cove noise further by about $3 \mathrm{~dB}$. The cove noise is slightly reduced by the decrease in the gap width, used as a noise reduction concept in the low noise HLFC configuration. For the gap noise component, the controlling parameter is the gap width, which explains the relatively unchanged noise levels for the first three configurations in Figure 11, which all have the same gap width. The low noise configuration, the fourth one in the table, assumes a $50 \%$ gap width reduction that results in about $1.5 \mathrm{~dB}$ reduction for the gap noise component. For the bracket noise, the increase in the strut length to support the larger Krueger device for the optimized HLFC wing suffers from about $1 \mathrm{~dB}$ noise increase. The noise reduction concept of aligning the brackets with the incoming flow shows the potential of $3 \mathrm{~dB}$ noise reduction. Lastly from Figure 11, it can be seen that when cavity noise is present, it is lower than the other components, mostly because its low frequency nature is less weighted in the calculation of EPNL.

\section{Application to HWB Aircraft}

The applications discussed in the previous section are meant to show the qualitative acoustic effects of employing Krueger devices in aircraft high lift systems, revealing parametric trends in noise levels of various components in response to changes in design parameters. The discussions are qualitative because no detailed aircraft design is considered. In this section, a more quantitative case will be considered for the HWB aircraft configuration. The needs for Krueger devices in HWB aircraft are similar to T+W aircraft, resulting from the needs to apply flow control technologies. One such configuration is discussed in Ref 3, which will be used here to study the effects of Krueger devices, in particular, their impact on the total HWB airframe noise and their relative importance compared with other airframe noise components such as landing gears and trailing edges.

The HWB design results from a comprehensive study [Ref 1], of which, the features that are relevant to noise are summarized here to facilitate the discussions. The design follows the best practices in aircraft design as well as incorporates potential technologies that are likely to mature in the next decade, namely, in the timeframe of NASA's 
$\mathrm{N}+2$ definitions. This allows the design to be practically feasible, to meet various mission requirements, and to achieve a good balance between various factors such as fuel savings and aerodynamic performance. In addition to the specific mission requirements, the design meets the Federal Aviation Authority (FAA) FAR Part 25 regulations and other conventional requirements that comprise the Aircraft Level Design Criteria (ALDC). The airplane has a medium capacity, long-range mission similar to the Boeing 767 or 787 models. The design specific mission requirements are documented in Ref 1, with the main characteristics of the aircraft summarized in Table 5.

Table 5 Airplane mission requirements.

\begin{tabular}{lll}
\hline \multicolumn{1}{c}{ Parameter } & Unit & Value \\
\hline Number of Passengers & - & 224 \\
Design Payload & $\mathrm{lb}$ & 50000 \\
Maximum Payload & $\mathrm{lb}$ & 90000 \\
Range with Design Payload & $\mathrm{nm}$ & 8000 \\
Maximum Takeoff Weight & $\mathrm{lb}$ & 412199 \\
Cruise Mach Number & - & 0.85 \\
Wing Span & $\mathrm{ft}$ & 213.3 \\
Reference Wing Area & $\mathrm{ft}^{2}$ & 8048 \\
Wing Aspect Ratio & - & 5.62 \\
Reference Thrust & $\mathrm{lb}$ & 92000 \\
Number of Engines & - & 2 \\
Engine Diameter D & $\mathrm{in}$ & 121 \\
Engine Position to Trailing Edge & $\mathrm{D}$ & 0.94 \\
\hline
\end{tabular}

The configuration has two turbofan engines mounted on the upper surface of the airframe structure, as illustrated in Figure 12. The airframe design consists of three adjacent payload cabins above a single cargo bay, forming the center body of the HWB airplane. This center body is wrapped with a large, swept wing form, from which, approximately conventional wings extend to form a single, large wing with an extended center body chord. Two outwardly canted vertical stabilizers are mounted on the aft, outboard corners of the center body with two turbofan engines mounted in between. The aerodynamic features of the design include a smoothly blended wing-body planform with varying wing chord length along the wing span. The wing cross-section airfoil camber and twist also vary along the span to provide an approximately elliptical lift distribution despite the wide chord variations. The large chord length at the center body results in low section lift coefficients, permitting greater thickness-to-chord ratios despite the Mach 0.85 cruise speed. The low center body section lift coefficients also permit reduced aft camber, providing pitch trim with the elevons faired at the design center of gravity. This design, however, has a disadvantage in acoustics in that it significantly reduces the circulatory flow under the airframe so that the local velocity at the main landing gear locations is almost the same as that of the free stream velocity. In contrast, for a conventional aircraft design, the local velocity at the main landing gear location is only about 80 percent of the free stream value. This results in a difference in noise levels of about $6 \mathrm{~dB}$ [Ref 24] and, as will be demonstrated in this section, leads to the relatively higher levels of main landing gear noise for the HWB aircraft.

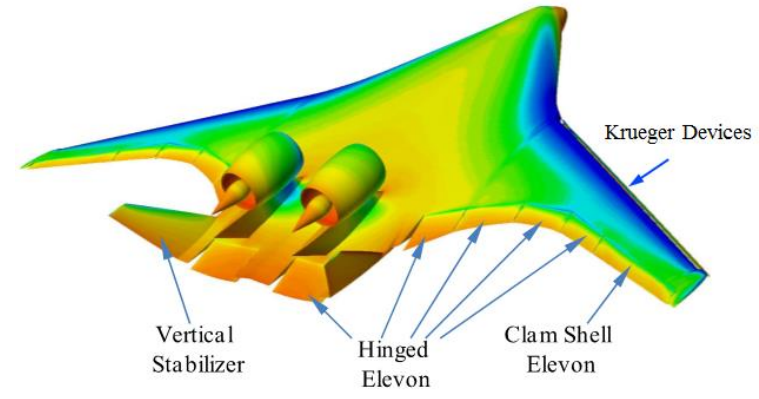

Figure 12. HWB aircraft configuration. 
As is well-known, HWB aircraft can provide sufficient lift at landing and takeoff conditions so that flaps are not needed, which is an acoustic advantage because flap side edges are known to be a major airframe noise source on conventional aircraft designs [Refs 25-27]. The HWB design has a system of trailing edge elevons and vertical stabilizers for flight control, as illustrated in Figure 12. Pitch control is provided by three simply hinged elevons at the trailing edge of the center body and two similar ones outboard on each side. There is also the potential to use symmetrical deflection of the vertical stabilizers to augment pitch control. Roll control is provided by deflection of the six trailing edge elevons on each outboard wing. The two inboard elevon surfaces are also used for pitch control. Deflection allocation of the six trailing edge devices is varied with airspeed to account for aero-elastic effects. Yaw control is provided by the rudders on the two vertical stabilizers. When additional yaw control power is needed, the rudders are augmented by the outboard two elevons on the wing, which can split into upper and lower portions, either deflecting together to act as an aileron or opening up apart to form a split trailing edge spoiler, providing drag control.

The HWB airframe has 12 sealed variable camber Krueger flaps, symmetrically designed about the airframe centerline with 6 on each side. The dimensions of the flaps are comparable to conventional designs, but their sweep angles are larger, due to the shape of the HWB platform. The sealed hinge Krueger flaps are also considered as an advanced noise reduction design. The acoustic effects due to the change from slotted slats to Krueger devices are discussed in the previous section, where it is shown that there are gains in some components while losses in others. The aggregate effects are not significant unless noise reduction treatments are applied. The sealed Krueger is one of the noise reduction concepts.

The landing gears are arranged in a tricycle configuration with a nose gear at the airframe centerline and two main gears at the junctions between the HWB center body and the outer wing. The nose gear has two wheels and the main gears have six-wheel tracks in three rows of two wheels each, similar to the main landing gears of the Boeing 777 aircraft. This six-wheel arrangement results in a narrower gear width, enabling the stowed gear to fit in the same depth as the lower deck cargo, compared with a four-wheel truck that requires increased depth, adversely affecting either center body thickness-to-chord ratio or center body chord length.

The airframe features discussed in the above paragraphs are the main sources of the HWB airframe noise. Their relative importance is illustrated in Figure 13, where the tone corrected perceived noise levels (PNLT) are plotted as a function of the observer time for the four components, namely, the Kruger device, the main landing gear, the nose landing gear, and the trailing edge devices. The noise levels are at approach conditions for aircraft noise certification, with the aircraft approaching for landing at 3-degree flight path 400 feet overhead at the microphone location. The flight speed is 146.1 knots, corresponding to a flight Mach number of 0.22 . The aircraft operates at an angle of attack of 11 degrees, and each engine's thrust is 3087 pounds.

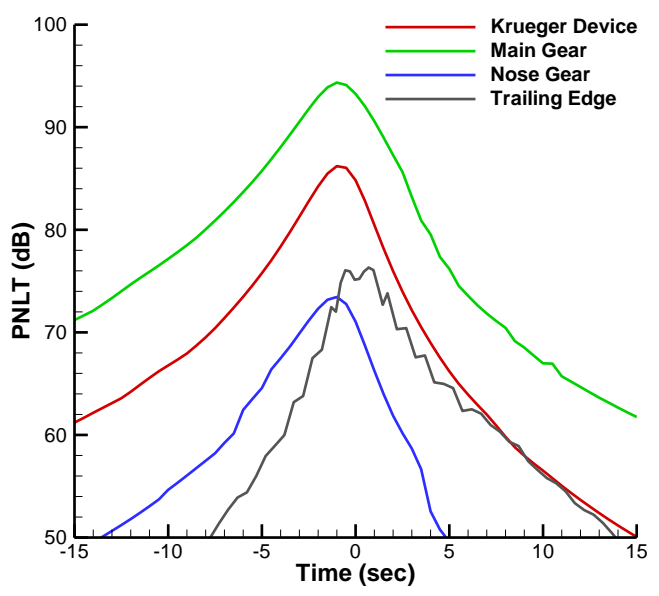

Figure 13. HWB airframe noise components.

The component noise levels shown in Figure 13 indicate the dominance of the main landing gear noise, followed by the Krueger device, and with the trailing edge noise and nose gear noise significantly lower. This can also be shown by EPNL, given in Figure 14. These trends can be readily explained by the unique characteristics of the HWB airframe. The high levels of the main landing gear noise are attributable to the high local flow velocity at the gear location and the large reflecting surface, as analyzed in detail in Ref 28. The Krueger noise benefits from the 
sealed gap, eliminating one of the four main sources associated with the Krueger device, as discussed in the previous section. The relatively low trailing edge noise is also expected because the trailing edge devices for the HWB aircraft are mostly for stability control without heavy loading. It should be pointed out that except for the sealed gap of the Krueger device, no other noise reduction concepts are assumed. Thus, the noise levels shown in Figure 13 and Figure 14 can be regarded as the baseline levels for this aircraft, and these levels can be potentially reduced with advanced noise reduction technologies.

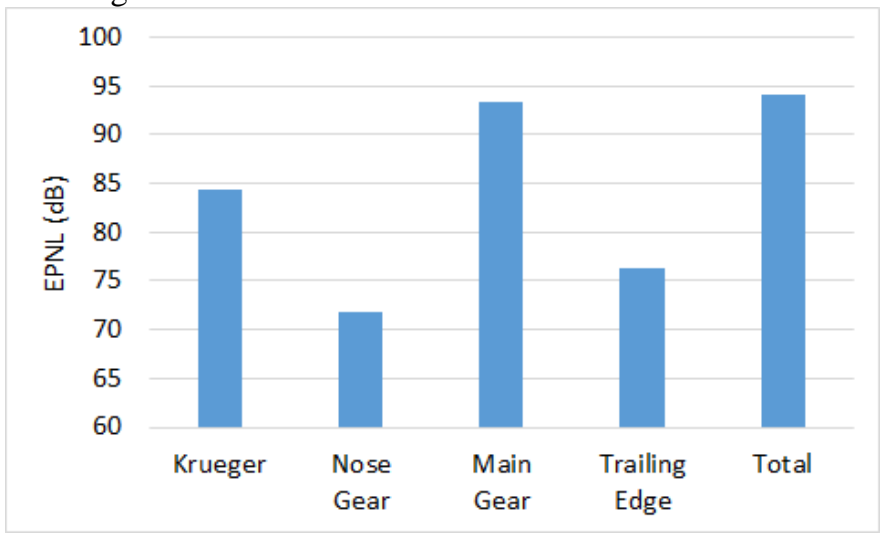

Figure 14. HWB airframe noise EPNL.

\section{Summary}

In this paper, the development of a prediction model has been presented for the Krueger noise component. The prediction methodology follows previously developed methods of physics-based modeling, capturing the dominant noise generation mechanisms and establishing correlations between noise and geometric and operational parameters. For the Krueger device, four noise sources have been discussed and modeled. They are the cove flow, the gap flow, the brackets and the cavity flow. Preliminary validations of the prediction model have been presented, showing good agreement between predictions and experimental data from a small scale wind tunnel test. Two examples have been discussed for the application of the prediction model, one for the conventional $\mathrm{T}+\mathrm{W}$ aircraft and another for the HWB aircraft. For the former, it has been shown that unless noise reduction technologies are utilized, the Krueger noise is comparable to slat noise for conventional slotted slats. In comparing the components between the two designs, it has been shown that the Krueger has lower cove flow noise, but the benefit is largely cancelled by its higher bracket noise and the new noise component from the cavity flow. For the application to the HWB aircraft that has a low noise design with a sealed Krueger, it has been shown that the total Krueger noise is lower than the main landing gear noise.

\section{Acknowledgments}

The authors would like to thank the NASA Environmentally Responsible Aviation Project for funding this effort. The authors would also like to thank Christopher Bahr and Florence Hutcheson of NASA LaRC for providing the Quiet Flow Facility test data for prediction model validation.

\section{References}

1 Bonet J. T., Schellenger H. G., Rawdon B. K., Elmer K. R., Wakayama S. R., Brown D. and Guo Y. P. "Environmentally Responsible Aviation (ERA) Project - N+2 Advanced Vehicle Concepts Study and Conceptual Design of Subscale Test Vehicle (STV)," NASA Contract Report, December 2011.

2 Guo Y. P., Nickol C. and Thomas R. H. "Noise and Fuel Burn Reduction Potential of an Innovative Subsonic Transport Configuration," AIAA Paper 2014-0257.

3 Guo Y. P., Burley C.L. and Thomas R. H. "On Noise Assessment for Blended Wing Body (BWB) Aircraft," AIAA Paper 2014-0365.

4 Thomas R. H., Burley C. L. and Olson E. D. "Hybrid Wing Body Aircraft System Noise Assessment with Propulsion Airframe Aeroacoustic Experiments," International Journal of Aeroacoustics, 11 (3+4), 369-410, 2012. 
5 Pott-Pollenske M., Almoneit D. and Wild J. "On the Noise Generation of Krueger Leading Edge Devices," AIAA Paper 2015-3142.

6 http://www.wired.com/images_blogs/autopia/2011/02/boeing7478I04-660x440.jpg, accessed on 10/22/2015.

7 Ffowcs Williams J. E. and Hawkings D. L. "Sound Generation by Turbulence and Surfaces in Arbitrary Motion," Phil. Tran. Roy. Soc. Lond. A264, 321, 1969.

8 Guo Y. P. "Aircraft Slat Noise Modeling and Prediction," AIAA Paper 2010-3837.

9 Guo Y. P. "A statistical model for landing gear noise prediction," Journal of Sound and Vibration 282, pp. 61-87, 2004.

10 Guo Y. P. "A Semi-Empirical Model for Aircraft Landing Gear Noise Prediction,” AIAA Paper 2006-2627, 2006.

11 Guo Y. P. "Aircraft Flap Side Edge Noise Modeling and Prediction," AIAA Paper 2011-2731.

12 Guo Y. P. "On Trailing Edge Noise Modeling and Prediction for Aircraft High Lift Wings" NASA Contract Report NASA Contract NNL07AA03A, 2010.

13 Bahr C. J., Hutcheson F. V., Thomas R. H. and Housman J. "Experimental Study of a Conventional Slat and a Krueger Flap High Lift Device," abstract submitted to the $22^{\text {nd }}$ AIAA/CEAS Aeroacoustics Conference, 2016.

14 Choudhari M., Khorrami M. R., Lockard D. P., Atkins H. L. and Lilley G. M. "Slat cove noise modeling: A posteriori analysis of unsteady RANS simulations," AIAA Paper 2002-2468.

15 Jenkins L. N., Khorrami M. R. and Choudhari M. "Characterization of unsteady flow structures near leadingedge slat: Part I. PIV measurements," AIAA Paper 2004-2801.

16 Khorrami M. R, Choudhari M. and Jenkins L. N. "Characterization of unsteady flow structures near leadingedge slat: Part II. 2D computations," AIAA Paper 2004-2802.

17 Guo Y. P. "A Discrete Vortex Model for Slat Noise Prediction,” AIAA Paper 2001-2157.

18 Dobrzynski W. and Pott-Pollenske M. "Slat Noise Source Studies for Farfield Noise Prediction," AIAA Paper 2001-2158.

19 Mendoza J. M., Brooks T. F. and Humphreys W. M. "Aeroacoustic Measurements of a Wing/Slat Model," AIAA Paper 2002-2604.

20 Guo Y. P. "Application of Ffowcs Williams/Hawkings Equation to Two Dimensional Problems," J. Fluid Mech. 403 pp201-221.

21 Ffowcs Williams J. E. “Hydrodynamic Noise,” Ann. Rev. Fluid Mech. 1, 197-222, 1969.

22 Crighton, D. G. "Basic Principles of Aerodynamic Noise Generation," Prog. Aerospace Sci. 16(1), 31-96, 1975.

23 Pott-Pollenske M., Alverez-Gonzalez J and Dobrzynski W. "Effect of Slat Gap on Farfield Radiated Noise and Correlation with Local Flow Characteristics," AIAA Paper 2003-3228.

24 Guo Y. P. "Effects of Local Flow Variations on Landing Gear Noise Prediction and Analysis," Journal of Aircraft 47, 383-391, 2010.

25 Guo Y. P. and Joshi M. "Noise Characteristics of Aircraft High Lift System," AIAA Journal, 41(7), 1247-1256, 2003.

26 Dobrzynski, W., Nagakura, K. Gehlhar, B. and Buschbaum, A. "Airframe Noise Studies on Wings with Deployed High-Lift System," AIAA Paper 1998-2337, June 1998.

27 Brooks T. F. and Humphreys W. M. "Flap edge aeroacoustic measurements and predictions," Journal of Sound and Vibration. 261, 31-74, 2003.

28 Guo Y. P., Burley C.L., and Thomas R. H. "Landing Gear Noise Prediction and Analysis for Tube-And-Wing and Hybrid-Wing-Body Aircraft," AIAA Paper 2016-1273, 2016. 